\title{
Perfil do suicídio em um estado do nordeste brasileiro
}

\author{
Suicide profile in a northeastern brazilian state \\ Perfil suicida en un estado brasileño del noreste
}

Recebido: 02/02/2021 | Revisado: 06/02/2021 | Aceito: 15/02/2021 | Publicado: 23/02/2021

\author{
Aline Mesquita Lemos \\ ORCID https://orcid.org/0000-0002-9242-6580 \\ Universidade Estadual do Ceará, Brasil \\ E-mail: alinemcastro@hotmail.com \\ Maria Salete Bessa Jorge \\ ORCID https://orcid.org/0000-0001-6461-3015 \\ Universidade Estadual do Ceará, Brasil \\ E-mail: maria.salete.jorge@gmail.com \\ Cybelle Façanha Barreto Medeiros Linard \\ ORCID: https://orcid.org/0000-0001-7927-9320 \\ Universidade Estadual do Ceará, Brasil \\ E-mail: cybellelinard@yahoo.com.br
}

\begin{abstract}
Resumo
O suicídio é um problema de saúde pública que perdura nos dias atuais no mundo todo. Objetivou-se descrever o perfil dos casos de suicídios no estado do Ceará. Utilizou-se uma abordagem quantitativa e descritiva, a coletada de dados foi realizada no sítio eletrônico do DATASUS, no recorte temporal de 2012 a 2019, nas estatísticas vitais dos grupos do banco de dados do Sistema de Informações sobre Mortalidade 1996 a 2015, pela CID-10 e dados preliminares de 2016 os dados sociodemográficos e o meio utilizado dos óbitos por suicídio no Estado do Ceará, assim como as estimativas populacionais do Instituto Brasileiro de Geografia e Estatística. Os dados foram analisados de acordo com a estatística descritiva, sendo fundamentada através de pesquisas das regiões e cidades brasileiras, com análise crítica dos dados. Os resultados demonstraram, no período compreendido entre 2012 a 2017, 3.393 óbitos por suicídio no Ceará, com a taxa de mortalidade por 100.000 habitantes. A distribuição dos óbitos provocados por suicídio, mostrou uma prevalência no sexo masculino, em indivíduos com idade compreendida entre 30 a 39 anos, com grau de escolaridade entre variando de 4 a 7 anos, solteiro, indivíduos pardos tendo como local principal de ocorrência o domicílio. Assim, são necessárias ações de saúde mental na promoção da saúde, prevenção de doenças e monitoramento da pessoa com risco para suicídio, estratégias que reforcem a sensibilização e empoderamento do profissional através da educação permanente nos serviços de saúde e sociedade civil principalmente no referente a perspectiva estigmatizada.
\end{abstract}

Palavras-chave: Suicídio; Indicadores de morbimortalidade; Causas externas.

\begin{abstract}
Suicide is a public health problem that persists today around the world. This study aimed to describe the profile of suicide cases in the state of Ceará. A quantitative and descriptive approach was used, data collection was performed on the DATASUS website, in the time frame from 2012 to 2019, in the vital statistics of the groups of the databases of the Mortality Information System 1996 to 2015, by the ICD-10 and preliminary data from 2016 the sociodemographic data and the means used death of deaths by suicide in the State of Ceará, as well as the population estimates of the Brazilian Institute of Geography and Statistics. The data were analyzed according to descriptive statistics, being based on research of Brazilian regions and cities, with critical analysis of the data. The results showed, in the period between 2012 and 2017, 3,393 deaths by suicide in Ceará, with the mortality rate per 100,000 inhabitants. The distribution of deaths caused by suicide showed a prevalence in males, in individuals aged between 30 and 39 years, with a level of education ranging from 4 to 7 years old, single, brown individuals with the main place of occurrence the household. Thus, mental health actions are needed in health promotion, disease prevention and monitoring of people at risk for suicide, strategies that reinforce the awareness and empowerment of professionals through continuing education in health services and civil society, especially with regard to the stigmatized perspective.
\end{abstract}

Keywords: Suicide; Morbidity and mortality indicators; External causes. 


\section{Resumen}

El suicidio es un problema de salud pública que persiste hoy en día en todo el mundo. Este estudio tenía como objetivo describir el perfil de los casos de suicidio en el estado de Ceará. Se utilizó un enfoque cuantitativo y descriptivo, la recopilación de datos se realizó en el sitio web de DATASUS, en el plazo de 2012 a 2019, en las estadísticas vitales de los grupos de las bases de datos del Sistema de Información de Mortalidad 1996 a 2015, por la CIE-10 y datos preliminares de 2016 los datos sociodemográficos y los medios utilizados de muerte por suicidio en el Estado de Ceará, así como las estimaciones de población del Instituto Brasileño de Geografía y Estadística. Los datos fueron analizados de acuerdo con estadísticas descriptivas, basándose en investigaciones de regiones y ciudades brasileñas, con análisis críticos de los datos. Los resultados mostraron, en el período comprendido entre 2012 y 2017 , 3.393 muertes por suicidio en Ceará, con la tasa de mortalidad por cada 100.000 habitantes. La distribución de las muertes causadas por el suicidio mostró una prevalencia en los varones, en individuos de entre 30 y 39 años, con un nivel de educación que oscilaba entre los 4 y los 7 años, individuos solteros y marrones con el principal lugar de ocurrencia del hogar. Así, se necesitan acciones de salud mental en la promoción de la salud, la prevención de enfermedades y el seguimiento de las personas en riesgo de suicidio, estrategias que refuercen la concienciación y el empoderamiento de los profesionales a través de la educación continua en los servicios de salud y la sociedad civil, especialmente en lo que respecta a la perspectiva estigmatizada.

Palabras clave: Suicidio; Indicadores de morbilidad y mortalidade; Causas externas.

\section{Introdução}

O suicídio está entre as principais causas de mortes no mundo para todas as idades. Por ano 800 milhões de pessoas morre por suicídio, ou seja, uma morte a cada 40 segundos. Essa taxa aumentou nos últimos 45 anos para $60 \%$ em todo o mundo, sendo uma das três principais causas de morte, do grupo de 15 a 44 anos em alguns países, e a segunda causa de morte no grupo de faixa etária de 15 a 29 anos. Enquanto a tentativa de suicídio ocorre com uma frequência em torno de 20 vezes que o suicídio consumado (Onu, 2016).

Segundo dados da Organização Panamericana de Saúde a pandemia provocada pelo coronavírus provocou um aumento aos fatores e risco para suicídio. Sendo a segunda causa de morte entre os jovens de 15 a 29 anos (Opas, 2021).

No Brasil, de acordo com dados do Departamento de Informática do Sistema Único de Saúde (DATASUS), foram contabilizadas um quantitativo de 195.979 mortes por suicídio no período compreendido entre 1996 e 2017 (Filho, 2019). No Ceará 519 pessoas chegaram ao suicídio no ano de 2020, segundo dados do Sistema de Informação integra SUS Ceará, da Secretaria de Saúde do Ceará (Ceará, 2020).

Na região Nordeste com o quantitativo de 2.540, sendo o Ceará com 565 óbitos por suicídio, com maior número com representação da região nordeste, ou seja, 22,2 \% dos casos (Brasil, 2016a). Em 2016 apresentou 10.575 casos de suicídio, sendo no Nordeste 2.470 e no Ceará 528 segundo o DATASUS em 2017 segundo Secretaria do Estado em 2017 consta 634.

O suicídio não é um ato aleatório ou sem finalidade, podendo ele representar a tentativa de saída de uma situação carregada de intenso sofrimento. É um tema complexo e que merece reflexões por parte de profissionais de várias áreas de atuação como enfermeiros, psicólogos, psiquiatras, antropólogos, sociólogos, entre outros. Vários aspectos envolvendo o suicídio tem sido motivo de interesse e investigação (Perreira et al., 2018).

Nesse contexto, é preocupante não somente o estigma ao longo dos anos na saúde mental, assim como a percepção depreciativa e negligência de alguns profissionais diante do problema e a influência destas no modo como a atenção em saúde é disponibilizada ao paciente. Com base no exposto, o estudo teve como objetivo descrever o perfil dos casos de suicídios no estado do Ceará.

\section{Metodologia}

O estudo caracteriza-se por ser do tipo descritivo com abordagem quantitativa. 
Gil (2008) refere que dentre as pesquisas descritivas pode-se destacar aquelas que têm como objetivo estudar as características de um grupo, levando em consideração a sua distribuição por idade, sexo, procedência, nível de escolaridade, estado de saúde física e mental dentre outras. A pesquisa científica constitui uma forma de melhor entendimento dos fenômenos e a possibilidade de acharmos respostas as constantes demandas que precisam ser resolvidas dentro da área do conhecimento (Pereira, et al, 2018).

A pesquisa foi realizada no sítio eletrônico do DATASUS no link acesso a informação no item informações de saúde (TABNET), tendo como recorte temporal o período compreendido entre 2015 a 2019, nas estatísticas vitais dos grupos do bancos de dados do Sistema de Informações sobre Mortalidade (SIM) 1996 a 2015, pela CID-10 e dados preliminares de 2016 os dados sociodemográficos e o meio utilizado dos óbitos por suicídio no Estado do Ceará, assim como as estimativas populacionais do Instituto Brasileiro de Geografia e Estatística (IBGE) . Além disso, para busca dos dados sociodemográficos de 2017, foi consultada via on-line as informações do site da Secretaria de Saúde do Estado do Ceará, que tinham somente dados referentes ao sexo e idade.

As informações buscadas no DATASUS referiam-se aos dados sociodemográficos e lesões autoprovocadas intencionalmente (suicídio) da Décima Revisão da Classificação Internacional de Doenças (CID-10) que corresponde categorias de X60 a X84, incluindo:

- sexo (masculino e feminino);

- idade ( 5 a 9,10 a 14,15 a 19,20 a 29,30 a 39,40 a 49,50 a 59 e 60 anos e mais);

-escolaridade (Nenhuma, 1 a 3, 4 a 7, 8 a 11, e 12 anos e mais);

-estado civil (solteiro, casado, viúvo, separado judicialmente e outro);

-local ocorrência (hospital, domicílio, viapública, outros e outro estabelecimento de saúde);

-cor/raça (branca, preta, amarela, parda e indígena); assim como o meio utilizado para o suicídio que foram categorizada segundo Carmo et al (2018) em:

- autointoxicação por medicamentos, substâncias biológicas e não especifcadas (X60-X64);

- autointoxicação intencional por álcool (X65);

- autointoxicação intencional por pesticidas e produtos químicos (X68-X69);

- lesão autoprovocada intencional por enforcamento e estrangulamento (X70);

- lesão autoprovocada intencional por afogamento/submersão (X71);

- lesão autoprovocada intencional por arma de fogo (X72-X74);

- lesão autoprovocada intencional por fumaça, fogo e gás (X75-X77);

- lesão autoprovocada intencional por arma branca e objetos contundentes (X78-X79);

- lesão autoprovocada intencional por precipitação de lugar elevado (X80);

- lesão autoprovocada intencional por meio não especifcado (X84);

- demais causas (X81-X83; X66-X67).

Os dados foram analisados de acordo com a estatística descritiva, sendo fundamentada através de pesquisas das regiões e cidades brasileiras, como análise crítica dos dados.

Por se tratar de um estudo de dados secundários disponíveis publicamente e sem identificação dos sujeitos, este não necessitou de submissão a Comitê de Ética em Pesquisa (CEP). No entanto foram respeitados e seguidos os preceitos éticos da Resolução do Conselho Nacional de Saúde (CNS) n 510, de 7 de abril de 2016 (Brasil, 2016b) e da Resolução 466/12 do 
Conselho Nacional de Saúde, que regulamenta a pesquisa envolvendo seres humanos onde estabelece os princípios éticos de autonomia, beneficência, não maleficência e de justiça (Brasil, 2012).

\section{Resultados e Discussões}

No período de 2012 a 2017, foram identificados 3.393 óbitos por suicídio no estado do Ceará, com a taxa de mortalidade por 100.000 habitantes que variou de 5,8 em 2012, com aumento em 2013 e decréscimo progressivo em 2014, 2015 e 2016, no entanto em 2017 aumentou para 7,2, ou seja, um crescimento de 25\% se compararmos 2012 e 2017.

A busca de justificativas para esse para este aumento da taxa de suicídio entre 2016 e 2017 pode estar atrelada ao aumento do índice de desemprego, aumento da violência e o momento político da conjuntura do país nesse período.

A distribuição dos óbitos provocados por suicídio entre os anos de 2015-2019, mostrou uma prevalência no sexo masculino, em indivíduos com idade compreendida entre 30 a 39 anos, com grau de escolaridade entre variando de 4 a 7 anos, solteiro, local principal de ocorrência é no domicílio e em indivíduos pardos (Tabela 1).

Tabela 1 - Distribuição dos óbitos por suicídio, de acordo com ano e características, Ceará, Brasil, 2015-2019.

\begin{tabular}{|c|c|c|c|c|c|c|c|c|c|c|}
\hline Variáveis & $\begin{array}{c}\begin{array}{c}2015- \\
T x=6,3\end{array}\end{array}$ & & $\begin{array}{c}\begin{array}{c}2016- \\
T x=6,5\end{array} \\
\text { T }\end{array}$ & & $\begin{array}{c}\text { 2017- } \\
T x=7,1\end{array}$ & & $\begin{array}{c}\begin{array}{c}2018- \\
T x=7,1\end{array}\end{array}$ & & $\begin{array}{c}\begin{array}{c}2019- \\
T x=6,7\end{array} \\
\end{array}$ & \\
\hline & $n=565$ & $\%$ & $\mathrm{n}=592$ & $\%$ & $n=644$ & $\%$ & $n=656$ & $\%$ & $n=628$ & $\%$ \\
\hline \multicolumn{11}{|l|}{ Sexo } \\
\hline Masculino & 440 & 77,9 & 487 & 82,3 & 534 & 82,9 & 539 & 82,2 & 496 & 79,0 \\
\hline Feminino & 125 & 22,1 & 105 & 17,7 & 110 & 17,1 & 117 & 17,8 & 132 & 21,0 \\
\hline \multicolumn{11}{|l|}{ Idade } \\
\hline 10 a 14 anos & 9 & 1,6 & 3 & 0,50 & 8 & 1,2 & 5 & 0,8 & 9 & 1,4 \\
\hline 15 a 19 anos & 41 & 7,25 & 41 & 6,92 & 49 & 7,6 & 48 & 7,3 & 54 & 8,6 \\
\hline 20 a 29 anos & 116 & 20,5 & 121 & 20,4 & 127 & 19,7 & 117 & 17,8 & 132 & 21,0 \\
\hline 30 a 39 anos & 118 & 20,9 & 129 & 21,8 & 140 & 21,7 & 136 & 20,7 & 134 & 21,3 \\
\hline 40 a 49 anos & 113 & 20,0 & 112 & 18,9 & 125 & 19,4 & 139 & 21,2 & 104 & 16,6 \\
\hline 50 a 59 anos & 76 & 13,45 & 84 & 14,2 & 88 & 13,7 & 94 & 14,3 & 95 & 15,1 \\
\hline $\begin{array}{c}60 \text { anos e } \\
\text { mais }\end{array}$ & 90 & 16,0 & 101 & 17,1 & 106 & 16,5 & 117 & 17,8 & 100 & 15,9 \\
\hline $\begin{array}{c}\text { Idade } \\
\text { ignorada }\end{array}$ & 2 & 0,4 & 1 & 0,2 & 1 & 0,2 & - & - & - & - \\
\hline
\end{tabular}




\section{Escolaridade}

\begin{tabular}{|c|c|c|c|c|c|c|c|c|c|c|}
\hline Nenhuma & 47 & 8,3 & 64 & 10,8 & 49 & 7,6 & 73 & 11,1 & 66 & 10,5 \\
\hline 1 a 3 anos & 141 & 25,0 & 137 & 23,1 & 162 & 25,2 & 120 & 18,3 & 103 & 16,4 \\
\hline 4 a 7 anos & 159 & 28,1 & 163 & 27,5 & 198 & 30,7 & 179 & 27,3 & 175 & 27,9 \\
\hline 8 a 11 anos & 79 & 14,0 & 88 & 15,0 & 101 & 15,7 & 132 & 20,1 & 138 & 22,0 \\
\hline $\begin{array}{c}12 \text { anos e } \\
\text { mais }\end{array}$ & 35 & 6,2 & 28 & 4,7 & 46 & 7,1 & 58 & 8,8 & 43 & 6,8 \\
\hline Ignorado & 104 & 18,4 & 112 & 18,9 & 88 & 13,7 & 94 & 14,3 & 103 & 16,4 \\
\hline \multicolumn{11}{|l|}{ Estado civil } \\
\hline Solteiro & 306 & 54,1 & 323 & 54,56 & 358 & 55,6 & 354 & 54,0 & 350 & 55,7 \\
\hline Casado & 147 & 26,0 & 147 & 24,83 & 168 & 26,1 & 177 & 27,0 & 152 & 24,2 \\
\hline Viúvo & 26 & 4,6 & 19 & 3,20 & 27 & 4,2 & 21 & 3,20 & 22 & 3,5 \\
\hline $\begin{array}{c}\text { Separado } \\
\text { judicialment }\end{array}$ & 27 & 4,8 & 28 & 4,72 & 25 & 3,9 & 35 & 5,33 & 28 & 4,5 \\
\hline Outro & 15 & 2,7 & 18 & 3,04 & 15 & 2,3 & 32 & 4,9 & 28 & 4,5 \\
\hline Ignorado & 44 & 7,8 & 57 & 9,62 & 51 & 7,9 & 37 & 5,64 & 48 & 7,6 \\
\hline
\end{tabular}

Local

ocorrência

\begin{tabular}{ccccccccccc}
\hline Hospital & 81 & 14,3 & 54 & 9,1 & 85 & 13,2 & 72 & 11,0 & 70 & 11,1 \\
Domicílio & 314 & 55,6 & 362 & 61,1 & 393 & 61,0 & 421 & 64,2 & 401 & 63,9 \\
Via pública & 50 & 8,8 & 35 & 6,0 & 26 & 4,0 & 35 & 5,3 & 25 & 4,0 \\
Outros & 112 & 19,9 & 133 & 22,5 & 131 & 20,3 & 117 & 17,8 & 125 & 19,9 \\
Outro & 3 & 0,5 & 6 & 1,0 & 7 & 1,1 & 6 & 0,9 & 7 & 1,1 \\
estab.de & & & & & & & & & & \\
saúde & & & & & & & & & & \\
Ignorado & 5 & 0,9 & 2 & 0,3 & 2 & 0,3 & 5 & 0,8 & - & - \\
\hline
\end{tabular}

Cor/raça

Branca

$68 \quad 12,0 \quad 49$

8,3

$71 \quad 11,0$

$95 \quad 14,5 \quad 101 \quad 16,0$ 


\begin{tabular}{ccccccccccc}
\hline Preta & 11 & 1,9 & 3 & 0,5 & 8 & 1,2 & 6 & 0,9 & 9 & 1,5 \\
Amarela & 2 & 0,4 & 2 & 0,3 & - & & - & - & - & - \\
Parda & 339 & 60,0 & 436 & 73,6 & 548 & 85,1 & 548 & 83,5 & 505 & 80,4 \\
Indígena & - & - & 1 & 0,2 & 1 & 0,2 & 1 & 0,2 & 2 & 0,3 \\
Ignorado & 145 & 25,7 & 101 & 17,1 & 16 & 2,5 & 6 & 0,9 & 11 & 1,8 \\
\hline
\end{tabular}

Fonte: Autores.

O sexo masculino apresenta um percentual mais elevado entre os indivíduos que cometem suicídio. De acordo com Lima e colaboradores (2013) os indivíduos acometidos por comportamentos suicidas apresentam as mais variadas características demográficas, econômicas, culturais, sociais e de estilo de vida. Pressupõe-se que as pressões sofridas, desde a infância, com indivíduos do sexo masculino contribuam para esse maior índice de suicídio.

O sexo masculino sobrepondo o feminino pode estar relacionada com a questão de gênero, do machismo cultural e dos métodos com que utilizam, pois, as mulheres ao tentar suicídio utilizam medicamentos e objetos não violentos, diferente de métodos como enforcamento e arma de fogo.

No Boletim epidemiológico do Ministério da saúde (Brasil, 2017), a tendência de gênero masculino tem sido evidenciada em um estudo sobre a taxa de mortalidade no Brasil de 2011 a 2015, no boletim epidemiológico da secretária de vigilância, com sexo masculino predominante, sendo quatro vezes mais prevalentes quando comparado ao feminino e crescimento em ambos os sexos, de 8,4 para 9,1 sexo masculino e 2,3 para 2,5 no feminino.

Várias ações, manuais e capacitações têm sido realizadas a nível mundial, assim como tem sido responsável pela redução das taxas de suicídio, entre elas podemos elencar na rede de atenção primária, secundária e demais órgãos de forma contínua a atenção integral do paciente com risco de suicídio.

Fazendo paralelo aos óbitos por suicídio no mundo, em 2015 houve em média 800.00 mortes com uma taxa de 10,7 por 100.000 habitantes, ou seja, o Ceará apresentou mais da metade da taxa mundial com correspondente de 6,3, sendo superior à do Brasil em 5,7, no entanto a OMS em seu relatório refere taxa de 6,3. Quando comparado à taxa brasileira de 2012 e 2017, representada por 5,3 e 5,4, o Ceará teve taxa superior, principalmente quanto ao ano de 2017, com aumento respectivo de 0,5 e 1,8 (OMS, 2014; Brasil, 2017).

No representativo de cálculo mundial sobre o suicídio em 2015, estima-se que é uma morte a cada 40 segundos, configurando $1,4 \%$ das mortes totais, e a $17^{\text {a }}$ principal causa de morte, sendo as tentativas 20 vezes mais freqüentes do que o próprio suicídio (OMS, 2016). Evento que prevalece em vários países e que necessita constantemente de sensibilização e formulação das políticas públicas.

O período de vida que apresenta um maior percentual de quantidade de suicídio é compreendido entre 30-39 anos. Maia-Silva e colaboradores (2021) mostraram que experiencias adversas durante a infância provoca um grande impacto na saúde dos adultos e em seus resultados sociais. Quando esse indivíduo chega à idade adulta e sofre pressões profissionais, familiares e pessoais e não possuiu uma infância equilibrada pode contribuir para o maior índice de suicídio nesse período da vida. 
Em um estudo realizado com profissionais de saúde através de grupo focal em um município no Ceará com a maior taxa de suicídio, foram associados os principais motivos para morte elencados como: falta de trabalho, ociosidade, alcoolismo, conflitos familiares e sofrimento psíquico (Oliveira, 2010).

Em países de baixa e média renda o suicídio foi responsável por $78 \%$ deste evento por adultos jovens e mulheres idosas, em contrapartida os de alta renda são homens de meia-idade, bastante representativo segundo relatório de 2015 (ONU, 2017).

Quanto aos idosos, a representação na proporcionalidade foi significante no Ceará, porém a OMS (2014) atrela que o suicídio em idosos com 70 anos ou mais é prevalente em quase todas as regiões do mundo, provavelmente fato este interligado a pessoa idosa a doenças, aposentadorias, pouca ênfase pela mídia e da saúde pública. Além disso, os idosos é um grupo de vulnerabilidade ao ato suicida, pois ele pode perpassar a intenção durante a vida não alcançado somente aos jovens (Cavalcante, et al,2015).

Observou-se que em torno de 28,3\% dos que cometeram suicídio tinham escolaridade de 4 a 7 anos, no entanto é importante perceber no referente 1 a 3 anos de escolaridade, ao longo do tempo tem decrescido, contrapondo aos que tem de 8 a 11 anos de estudo.

Machado e Santos (2015) também encontrou em seus estudos sobre mortalidade brasileira por suicídio entre os anos de 2000 e 2012 o risco maior de suicídio em pessoas que tinham de 1 a 7 anos de estudos com proporção de 77,5\% e 63\% respectivamente.

Em estudos realizados na Bahia caminham no mesmo sentido, e apontam maior número de suicídios em pessoas com menos de 7 anos de escolaridade (Carmo et al, 2018, Souza, 2011).

De acordo com o estado civil que se destacou como risco para suicídio foi a proporção de solteiros (52,5\% a 59,7 \%) quando em comparação aos casados ( $25 \%$ a $29 \%$ ), ou seja, duas vezes mais propensos do que as pessoas casadas. Se somarmos solteiros, viúvos e separados judicialmente encontramos aproximadamente $61 \%$.

Corroborando com Ribeiro et al (2018), em seu estudo sobre os suicidas 2001 a 2013 no Piaú, encontramos a mesma proporção no que diz respeito ao quantitativo equivalente de solteiros com 53,7\% e casados com 32,7\%, da mesma forma se somarmos e considerarmos as três categorias citadas acima constatamos o mesmo percentual de $61 \%$.

Em contrapartida em um estudo realizado em 2006 na cidade de Itabira, em 1990 e 2001 constatou-se que os suicidas metade eram casados e a outra solteiros (Souza; Minayo; Cavalcante, 2006).

No correspondente ao local da ocorrência em todos os anos no Ceará, a maioria dos casos se concentra em domicílio com porcentagem maior em 2016 com $64 \%$, seguido de outros com 21,6\%, e taxas menor do que $8 \%$ em hospital, via pública e outro estabelecimento de saúde.

A tendência do suicídio quanto ao lugar, por exemplo, em Brasília constatou-se na maioria das vezes suicídio na própria residência (48,4\%) e em Amazonas e Manaus esse valor foi maior ainda com 77,4 \% e 75,4 \% no referente a suicídios de 2005 a 2009 (Silva, 2016; Orellana, Basta; Souza, 2013).

Por problemas de transtornos mentais, sendo o mais comum a depressão, trazendo consigo o isolamento, ser solteiro ou residir só, parece ter relação no propiciamente do suicídio em pessoas no seu próprio domicílio.

Em relação ao campo raça/cor foram encontrados com maior número em 2016 (548 pardos) com discreta diminuição em 2019, e de forma ignorada que iniciou em 2015 com 25,7\% dos casos e em 2016 reduziu para 1,8\%.

Em 2015, de acordo com o último censo relativo aos dados da Pesquisa Nacional por Amostra de Domicílios (PNAD), as pessoas se declararam brancos $(45,22 \%)$, pardos $(45,06 \%)$, pretos $(8,86 \%)$, amarelos $(0,47 \%)$ e $(0,38 \%)$ como indígenas (IBGE, 2018). 
Quanto ao suicídio segundo boletim epidemiológico do Brasil segundo raça/cor, mostrou que 49,6\% das mulheres eram brancas e 35,7\%, negras (pardas + pretas) e no sexo masculino $49 \%$ e 37,2\% respectivamente, diferente da tendência no Ceará (Brasil, 2017).

Em contrapartida em uma pesquisa realizada no Brasil no sobre perfil do suicida de 2000 a 2012, os indígenas apresentaram a maior taxa de mortalidade com 8,6 e 14,4 por 100.000, maior do que a taxa nacional em $132 \%$, as sim como do crescimento de 68,7\% ao longo dos 12 anos supracitados (Machado; Santos, 2015). Estando prevalente também de 2011 a 2015 na população indígena em geral (15,2/100 mil hab.) e no sexo masculino (23,1/100 mil hab.) na maioria com adolescentes $(44,5 \%)$ de 10 a 19 anos.

Para indígenas e não indígenas em Roraima em um estudo de 2009 a 2013, as taxas de mortalidade geral foram de 15,0 e 8,6, quando do sexo masculino essa taxa é maior ainda contabilizando 20,3 e 12,9, no entanto a proporção do total de óbitos foi de 17,1\% e 82,9\% entre indígenas e não indígenas (Costa; Onety Júnior, 2017).

Quanto à raça indígena percebe-se que no Ceará não é prevalente, no entanto em nível de Brasil o quantitativo é o mais representativo quanto ao suicídio, é o que concerne às pesquisas nos dados de 2000 a 2015 (Brasil, 2017).

Sobre a vertente do aumento de casos em 2017 no Ceará, quando comparamos 2016 e 2017, pode ser um viés da pesquisa, que pode ser provavelmente uma maior notificação de casos e um melhor preenchimento das D.O, assim como também treinamentos e incentivos de sensibilização na questão da notificação, já que existe um processo de estigmatização sobre suicídio.

A incompletude dos dados da Tabela 1, principalmente relacionado ao item ignorado como, por exemplo, no ano de 2016, no relativo à questão da idade $(0,2 \%)$, idade $(20,6 \%)$, estado civil $(10,6 \%)$, local de ocorrência $(0,4 \%)$ e principalmente no correspondente a cor $(13,6 \%)$ são reflexos que apresenta limitações como as questões de falhas no preenchimento das declarações de óbitos, assim como provavelmente uma subnotificação no sentido de sub-registro. Quando a causa é classificada como externa por intenção indeterminada ou mal definida envereda-se a questão de subnotificação e dificulta para uma melhor fidelização dos casos.

A distribuição dos óbitos por suicídio de acordo com a categoria do CID-10 apresenta como maior percentual as lesões provocadas intencionalmente por enforcamento e estrangulamento. Correspondendo a um percentual de 79,3\% e 77,4\% nos anos de 2018 e 2019 respectivamente (Tabela 2).

Lesão autoprovocada intencional por enforcamento e estrangulamento (X70). 
Tabela 2 - Distribuição dos óbitos por suicídio, de acordo com as categorias da CID-10a, Ceará, Brasil, 2015-2019.

\begin{tabular}{llllllllllll} 
Ano & 2015 & & 2016 & & 2017 & & 2018 & & 2019 \\
\hline & n & $\%$ & n & $\%$ & n & $\%$ & n & $\%$ & n & $\%$
\end{tabular}

Autointoxicação por

medicamentos, substâncias

biológicas e não especificadas

(X60-X64);

$\begin{array}{llllllllll}30 & 5,3 & 22 & 3,71 & 25 & 3,88 & 28 & 4,3 & 23 & 3,7\end{array}$

Autointoxicação intencional por álcool (X65);

Autointoxicação intencional por pesticidas e produtos químicos (X68-X69);

Lesão autoprovocada intencional por enforcamento e estrangulamento (X70);

Lesão autoprovocada intencional por afogamento/submersão (X71);

Lesão autoprovocada intencional por arma de fogo (X72-X74);

Lesão autoprovocada intencional por fumaça, fogo e gás (X75-X77); Lesão autoprovocada intencional por arma branca e objetos contundentes (X78-X79);

Lesão autoprovocada intencional por precipitação de lugar elevado (X80);

Lesão autoprovocada intencional por meio não especifcado (X84); Demais causas gases/obj mov./impac.veiculo/meios espec.

(X81-X83; X66-X67) $1 \quad 0,1$

Total 565 592 644 656 628

X75 e x77 não tem nos dados. Fonte: Autores.

No Brasil de 1980 a 2006, 2000 a 2012 e de 2011 a 2015 que diz respeito aos métodos usados para o suicídio, o enforcamento lidera na proporcionalidade seguido de intoxicação exógena (pesticida) e arma de fogo (LOVISI, 2009; Machado; Santos, 2015; Brasil, 2017). 
Em estudo realizado por Gomes, Araújo e Gomes (2018), mostrou que a taxa de suicídio entre militares é alta, sendo a idade mais jovem e o posto militar mais baixo preditores independentes de suicídio nesta subpopulação. Fato como depressão, ansiedade podem agravar a ideação suicida e o óbito provocado pelo suicídio.

Na lista de doenças e agravos de notificação compulsória foi integrado no SINAN em 2011 a notificação de violências interpessoais e autoprovocadas em todos os serviços de saúde, seja ele público ou privado no Brasil. Agressão, automutilação e tentativa de suicídio é classificada como violência autoprovocada, com exceção daquelas que tenha como desfecho o óbito.Um avanço em 2014, surgiu quando a pessoa com tentativa de suicídio, tornou-se notificação obrigatória onde a Secretaria Municipal de Saúde tem que notificar até 24 horas, respaldado pela portaria do Ministério da Saúde ${ }^{\circ}$ 1.271/2014. Com isso o acompanhamento de emergência e psicossocial na rede integrada, interliga informações relacionadas ao paciente consequentemente número de notificações e sua trajetória na rede de cuidado, evitando assim muitas vezes o próprio suicídio (Brasil, 2016c).

A declaração de óbito também constitui um instrumento diretivo para estatísticas plausíveis, assim como outros dados deste documento como profissão e bairro podem compor o sistema DATASUS, no intuito de facilitar pesquisas e construções de políticas públicas com ações. Constitui-se possivelmente uma limitação do estudo o preenchimento inadequado da declaração de óbito.

Espera-se que esta pesquisa possa contribuir para a comunidade científica e aos profissionais de saúde, ao gerar informações acerca das características e fatores que se associam à ocorrência de suicídio. Bem como, promover reflexões e mudanças no planejamento de intervenções educativas, preventivas e assistenciais aos pacientes que manifestem ideação suicida.

A educação em saúde nos serviços urge para orientação da população, tanto na detecção como na questão do estigma, principalmente quando do quadro de depressão ou outro transtorno mental e eventos estressores. Portanto, é preciso dar continuidade e fortalecimento das estratégias nos diversos níveis de atenção em saúde, de forma integral e em rede, assim como promover ações de políticas públicas nos municípios como promoção da saúde e prevenção de agravos reluzente a pessoa com ideia suicida.

As capacitações de educação permanente de notificação dos casos de violência ou lesões autoprovocadas, se fazem necessário para os profissionais de saúde para que sejam sensibilizados, assim como dos sinais de alerta do suicídio, que muitas vezes se revelam principalmente no âmbito familiar.

\section{Conclusão}

A partir dos dados apresentados conclui-se que de 2015 a 2019 no Ceará, o sexo, faixa etária, estado civil, escolaridade, cor, local da ocorrência e recurso utilizado demonstraram-se associados ao suicídio com indivíduos do sexo masculino, de 30 a 39 anos, com grau de escolaridade de 4 a 7 anos, de cor parda, que utilizam o domicílio como local através do método de enforcamento e estrangulamento.

Nossas pesquisas continuam e temos interesse em continuar contribuindo com a sensibilização dos profissionais da saúde e educação.

\section{Referências}

Brasil. (2016a). Ministério da Saúde. Secretaria de Vigilância em Saúde. Departamento de Vigilância de Doenças e Agravos Não Transmissíveis e Promoção da Saúde. Viva: instrutivo notificação de violência interpessoal e autoprovocada [Internet]. 2. ed. Brasília: Ministério da Saúde, 92p. http://bvsms.saude.gov.br/bvs/publicacoes/viva_instrutivo_violencia_interpessoal_autoprovocada_2ed.pdf 
Brasil. (2016b). Ministério da Saúde, Conselho Nacional de Saúde. Resolução 510, de 7 de $\quad$ abril de 2016. https://bvsms.saude.gov.br/bvs/saudelegis/cns/2016/res0510_07_04_2016.html.

Brasil. (2016c). Ministério da Saúde. Departamento de Informação do SUS (DATASUS). Informações de saúde. Óbitos por causas externas [Internet]. http://tabnet.datasus.gov.br/cgi/tabcgi.exe?sim/cnv/ext10uf.def

Carmo, E. A, Santos, P. H. S, Ribeiro, B. S, et al. (2018). Características sociodemográficas e série temporal da mortalidade por suicídio em idosos no estado da Bahia, 1996-2013. Epidemiol. Serv. Saúde, 27(1), e20171971. https://www.scielo.br/pdf/ress/v27n1/2237-9622-ress-27-01-e20171971.pdf.

Cavalcante, A. C. S, Sérvio, S. M. T, Franco, F. R. A, Cunha, V. P, Cavalcante, F. V. \& Nascimento, C. E. M. (2011). A clínica do idoso em situação de vulnerabilidade e risco de suicídio. Revista Trivium Est. Interd - Estudos Interdisciplinares, 7(1), 74-87 http://dx.doi.org/10.18370/2176-4891.2015v1p74. http://pepsic.bvsalud.org/pdf/trivium/v7n1/v7n1a08.pdf.

Ceará (2020). Mortalidade por suicídio. https://indicadores.integrasus.saude.ce.gov.br/indicadores/indicadores-vigilancia-saude/situacaoepidemiologica/mortalidade-suicidio

Costa, A. L. S. da. \& Souza, M. L. P. (2017). Caracterização da mortalidade por suicídio entre indígenas e não indígenas em Roraima, Brasil, $2009-2013$. Revista Epidemiol. Serv. Saúde, 26(4), 887-893. https://www.scielo.br/pdf/ress/v26n4/2237-9622-ress-26-04-00887.pdf

Filho, D. B. F. (2019). O que dizem os números sobre suicídio no Brasil. Revista Questão de Ciência. Setembro. https://www.revistaquestaodeciencia.com.br/index.php/artigo/2019/09/20/o-que-dizem-os-numeros-sobre-suicidio-no-brasil.

Gil, A. C. (2008). Como elaborar projetos de Pesquisa. (4a ed.), Atlas.

Gomes, D. A. R., Araújo R. M. F. \& Gomes, M. S. (2018). Incidence of suicide among military police officers in South Brazil - An 11 year retrospective cohort study. Revista Compr Psychiatry, 85, 61-6. http://dx.doi.org/10.1016/j. comppsych.2018.06.006. PMid:29981945. https://pubmed.ncbi.nlm.nih.gov/29981945/\#: :text=The\%20present\%20study\%20aimed\%20to,PO\%20subpopulation\%20in\%20South\%20Brazil.\&text=A\% 20total $\% 20$ of $\% 20650 \% 20$ participants,cumulative $\% 20$ incidence $\% 20$ of $\% 20138 \% 2 \mathrm{~F} 100 \% 2 \mathrm{C} 000$.

IBGE. (2018) https://teen.ibge.gov.br/sobre-o-brasil/populacoa/cor-ou-raca.html.

Lima, N. N, Nascimento, V. B, Carvalho, S. M, Abreu, L. C, Neto, M. L, Brasil, A. Q, et al. (2013). Childhood depression: a systematic review. Neuropsychiatr Dis Treat, v. 2013(9), 1417-25. http://dx.doi.org/10.2147/NDT.S42402. Childhood depression: a systematic review (nih.gov).

Lovisi, G. M, Santos, S. A, Legay, L, Abelha, L. \& Valencia, E. (2009). Análise epidemiológica do suicídio no Brasil entre 1980 e 2006. Rev. Bras. Psiquiatria, 31(2), S86-S93. https://www.scielo.br/pdf/rbp/v31s2/v31s2a07.pdf.

Machado, D. B. \& Santos, D. N. dos. (2015). Suicídio no Brasil, de 2000 a $2012 . \quad J . \quad$ Bras. Psiquiatr., 64(1), 45-54. https://www.scielo.br/pdf/jbpsiq/v64n1/0047-2085-jbpsiq-64-1-0045.pdf.

Maia-Silva, K. M, Zamei, N, Selby, P, Fontes, C. J. F. \& Santos, U. P. (2021). Tobaco smoking associated with adverse childhood experiences in a Brazilian Community university sample: A case-control study. Children and Youth Services Review, 120, 105438. https://doi.org/10.1016/j.childyouth.2020.105438.

Oliveira, M. A. (2010). O suicídio no estado do Ceará: estudo de epidemiologia ecológica. Mestrado Acadêmico em Saúde Pública. Dissertação de mestrado, 101p. http://www.uece.br/ppsac/wp-content/uploads/sites/37/2011/03/MARLUCE-ALVES-DE-OLIVEIRA.pdf.

OMS - Organização Mundial da Saúde. Banco de dados de mortalidade. (2014). http://apps.who.int/healthinfo/statistics/mortality/whodpms.

ONU - Organização das Nações Unidas. (2017). Uma pessoa morre por suicídio a cada 40segundos, afirma OMS. https://www.paho.org/bra/index.php?option=com_content\&view=article\&id=6017:suicidio-uma-pessoa-morre-a-cada-40-segundos-afirma-oms\&Itemid=839.

ONU - Organização das Nações Unidas. Centro de Notícias da ONU. (2016). Unmillón de personas se suicidan cada año, según OMS. <http://www.who.int/mediacentre/factsheets/fs398/es/>.

OPAS, (2021). https://www.paho.org/pt/noticias/10-9-2020-pandemia-covid-19-aumenta-fatores-risco-para-suicidio.

Orellana, J. D. Y, Basta, P. C. \& Souza, M. L. P. (2013). Mortalidade por Suicídio: um enfoque em municípios com alta proporção de população autodeclarada indígena no Estado do Amazonas, Brasil. Rev. Bras. Epidemiol, 16(3), 658-669. https://www.scielo.br/pdf/rbepid/v16n3/pt_1415-790X-rbepid16-03-00658.pdf.

Pereira, A. S. et al. (2018). Metodologia da pesquisa científica. UFSM. https://repositorio.ufsm.br/bitstream/handle/1/158824/Lic Computação MetodologiaPesquisa-Científica.pdf?sequence $=1$

Perreira, A. S, Wilhelm, A. R, Koller, S. H. \& Almeira, R. M. M. (2018). Fatores de risco e proteção para tentativa de suicídio na adultez emergente. Ciência e Saúde Coletiva. 23(11), 3767-3777. 10.1590/1413-812320182311. 29112016. 1413-8123-csc-23-11-3767.pdf (scielo.br).

Ribeiro, J, Mascarenhas, T, Araújo, A, Coelho, D, Branca, S. \& Coelho, D. (2018). Perfil sociodemográfico da mortalidade por suicídio. Revista de Enfermagem UFPE on line, 12(1), 44-50. https://doi.org/10.5205/1981-8963-v12i1a25087p44-50-2018.

Silva, A. G. A. Registros de suicídio no Distrito Federal de 2000 a 2014. (2016). 33 f., il. Trabalho de conclusão de curso (Bacharelado em Enfermagem) Universidade de Brasília, Ceilândia-DF. 2016_AnaGabrielleAraujoeSilva.pdf (unb.br).

Souza, E, Minayo, M. C. de S. \& Cavalcante, F. G. (2006). O impacto do suicídio sobre a morbimortalidade da população de Itabira. Revista Ciência \& Saúde Coletiva, 11(10), 1333-1342. https://www.scielo.br/pdf/csc/v11s0/a22v11s0.pdf. 
Research, Society and Development, v. 10, n. 2, e45410212598, 2021

(CC BY 4.0) | ISSN 2525-3409 | DOI: http://dx.doi.org/10.33448/rsd-v10i2.12598

Souza, V. dos S, Alves, M. da S, Silva, L. A, Lino, D. C. S. F. Nery, A. A. \& Casotti, C. A. (2011). Tentativas de suicídio e mortalidade por suicídio em um município no interior da Bahia. J. Bras. Psiquiatr., 60(4), 294-300. https://www.scielo.br/pdf/jbpsiq/v60n4/a10v60n4.pdf. 